\title{
Increase of macrophage migration inhibitory factor in sera of patients with iridocyclitis
}

\author{
Nobuyoshi Kitaichi, Satoshi Kotake, Yuka Mizue, Hidehiko Matsuda, Kazunori Onoé, \\ Jun Nishihira
}

\begin{abstract}
Aims-To determine whether macrophage migration inhibitory factor (MIF) levels were increased in sera of the patients with iridocyclitis.

Methods-Sera were obtained from 41 patients with acute iridocyclitis, 13 patients with chronic iridocyclitis, and 44 healthy control subjects. MIF levels were determined by a human MIF ELISA.

Results-The average levels of MIF in the sera of patients with both acute and chronic iridocyclitis were significantly higher than that of healthy subjects. Conclusion-Uveitis induces the elevation of serum MIF, which may affect various inflammatory symptoms in uveitis. (Br F Ophthalmol 2000;84:1423-1425)
\end{abstract}

Department of Ophthalmology, Hokkaido University Graduate School of

Medicine

N Kitaichi

S Kotake

H Matsuda

Central Research Institute, Hokkaido University School of Medicine

J Nishihira

Sapporo

Immunodiagnostic

Laboratory, Co, Ltd

Y Mizue

Division of

Immunobiology,

Institute for Genetic

Medicine, Hokkaido

University

K Onoé

Correspondence to: Dr Kazunori Onoé, Division of Immunobiology, Institute for Genetic Medicine, Hokkaido University, Kita-15, Nishi-7, Kita-ku, Sapporo 060-0815, Japan kazunori@imm.hokudai.ac.jp

Accepted for publication 16 June 2000
Macrophage migration inhibitory factor (MIF) was reported as the first cytokine produced by activated T cells. ${ }^{1}$ Although it has been considered that production of MIF is associated with the generation of cell mediated immune responses, the biological role of MIF in vivo has been unclear for a long time. ${ }^{2}$ Recently we reported that MIF was involved in the signal pathway via $\mathrm{T}$ cell receptor (TCR) in T cells, ${ }^{3}$ and that the serum MIF levels of the patients with Behçet's disease, sarcoidosis, and VogtKoyanagi-Harada's (VKH) disease were significantly elevated compared with those of healthy control subjects. ${ }^{4}$ However, since these diseases cause not only ocular inflammation but also systemic inflammation, it has been unclear whether high levels of serum MIF reflected merely uveitis or systemic inflammation. MIF is a major secretory protein released by anterior pituitary cells, and potentiating lethality of endotoxin shock. ${ }^{5}$ In addition, it has been reported that MIF is a glucocorticoid induced immunomodulator for other proinflammatory cytokines. ${ }^{6}$ Although cytokines play an important part in ocular inflammation, ${ }^{78}$ it remains to be seen if the cytokines can be substantially produced in oculus, an immunologically privileged site, and contribute to the serum level. In the present study we analysed the serum MIF levels in patients with iridocyclitis who have anterior segment in- traocular inflammation but no systemic inflammation.

\section{Patients and methods}

After informed consent was obtained, sera were obtained from 41 age and sex matched patients with acute iridocyclitis (13 patients have HLA-B27), 13 patients with chronic iridocyclitis, and 44 healthy control subjects. Acute cases of iridocyclitis had a sudden onset which lasted up to 8 weeks. Chronic forms of iridocyclitis had an insidious onset and lasted longer than 8 weeks. Systemic symptoms as seen in ankylosing spondylitis (AS) and other collagen diseases were not complicated in these patients. Infectious iridocyclitis, Fuch's heterochromia, and Posner-Schlossman syndrome were not included in this study. Blood samples were collected at the first medical examination in the uveitis survey clinic of Hokkaido University Hospital. All patients were in active phase of iridocyclitis when blood samples were collected. All of the patients with acute and chronic iridocyclitis were given topical corticosteroids at this time. MIF levels were determined by a human MIF enzyme linked immunosorbent assay (ELISA) (Cosmobio, Tokyo, Japan) as described previously. ${ }^{9}$ Statistical analysis was performed using the MannWhitney U test. This study was performed according to the tenets of the Declaration of Helsinki.

\section{Results}

The mean MIF concentrations in the sera of patients with acute iridocyclitis, idiopathic chronic iridocyclitis, and healthy control subjects were 28.8 (SD 3.4), 18.5 (5.9), and 4.4 (0.2) $\mathrm{ng} / \mathrm{ml}$, respectively. The average level of MIF in the sera of iridocyclitis patients was significantly higher $(\mathrm{p}<0.0001)$ than that of healthy control subjects (Fig 1). No significant difference was found in the serum MIF level between the patients with acute iridocyclitis and those of chronic iridocyclitis. However, since only 13 samples were evaluated from the chronic group in the present study, the two high MIF samples might significantly alter the distribution. Examination of more samples from cases of chronic iridocyclitis is needed to 


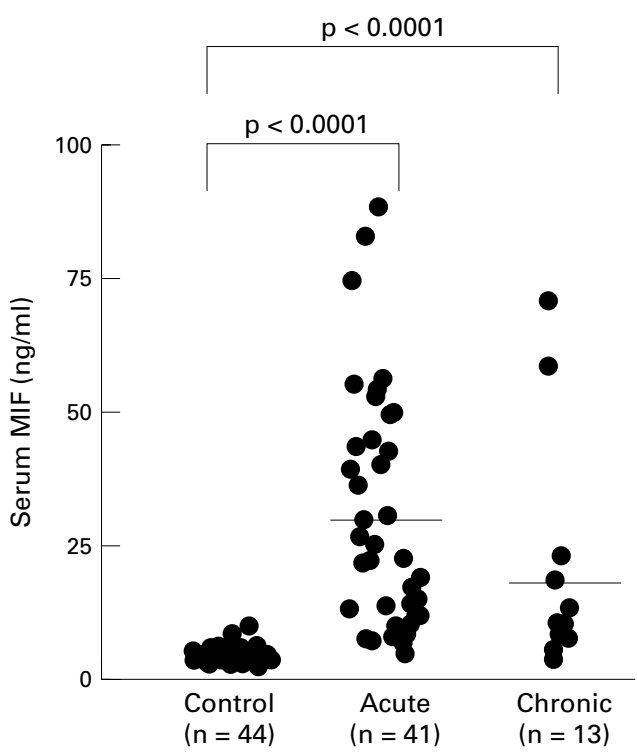

Figure 1 Concentration of MIF in sera of patients with acute and chronic iridocyclitis. The mean MIF concentration in the sera of patients with acute and chronic, iridocyclitis and of healthy control subjects are 28.8 (SD 3.4), 18.5 (5.9), and 4.4 (0.2) $n g / m l$, respectively. The average level of MIF in sera of patients with iridocyclitis is significantly higher than that of healthy control subjects $(p<0.0001)$

draw a conclusion on this matter. We divided the acute cases into two groups, on the basis of HLA-B27 haplotype. No significant difference was detected in the serum MIF level between the patients with HLA-B27 ${ }^{+}$(34.9 (6.6) $\mathrm{ng} / \mathrm{ml})$, and those with HLA-B27 ${ }^{-}$(25.9 (4.0) $\mathrm{ng} / \mathrm{ml})$.

\section{Discussion}

In the present study, we detected high levels of MIF in the sera of patients with iridocyclitis. We previously reported the prominent increase of MIF in the sera of patients with Behçet's disease, sarcoidosis, and VKH disease. ${ }^{4}$ Further, we found that patients with Behçet's disease in the ocular exacerbation stage showed higher levels of MIF than those in the convalescent stage. Nevertheless, the relation between increased MIF productivity and genetic background has been unclear. The investigation of genetic factors which affect the productivity of MIF may contribute to understanding the role of MIF. HLA-B27 is associated with acute iridocyclitis and AS. ${ }^{10}{ }^{11}$ In the present study, we found no significant difference in the serum MIF level between HLA-B27 positive and negative groups of patients. Since no patients had radiological evidence and clinical symptoms of AS, it seems that increased serum MIF seen in these patients is attributed to ocular inflammation, but not to HLA-B27 or AS.

There were several patients with Behçet's disease who showed in excess of $100 \mathrm{ng} / \mathrm{ml}$ of MIF in our previous study. ${ }^{4}$ However, the source of serum MIF was unclear. Since the space of oculi is limited, we considered that MIF production in the local lesion would have been quite low and the serum MIF might have been secreted by vascular endothelial cells in organs other than eyes in Behçet's disease.
In the present study, we detected considerably high levels of serum MIF in the patients with iridocyclitis who showed no systemic inflammation. This finding suggests three possible origins of MIF in patients with uveitis. The first source may be the uvea of these patients, including those with Behçet's disease and sarcoidosis. In previous studies, we observed that high levels of MIF were present in uvea $^{12}$ and vascular endothelial cells. ${ }^{13}$ Thus, it seems highly likely that a various cells in the oculus are responsible for MIF production. The second source may be the pituitary gland that is stimulated by ocular inflammation and releases MIF in the serum. In the murine model, it was reported that MIF circulated normally as a hormone and was released in part by the pituitary gland in response to stress or systemic inflammatory stimuli. ${ }^{2}{ }^{6}$ The third source may be peripheral blood mononuclear cells (PBMC). Recently, Shimizu et al reported that MIF production by PBMC was markedly upregulated in patients with atopic dermatitis. ${ }^{14}$ It seems that MIF produced in ocular tissues of iridocyclitis are in some way related to the systemic production of MIF via the pituitary gland, PBMC, and other cells even though apparent systemic inflammatory symptoms are not observed in patients with iridocyclitis. We would like to emphasise here the close association between uveitis and MIF in humans.

We and Bacher et al reported that anti-MIF $\mathrm{Ab}$ inhibited $\mathrm{T}$ cell proliferative responses in vitro. $^{315}$ Furthermore, we recently reported that anti-MIF mAb administration inhibited experimental autoimmune uveoretinitis (EAU) in the murine model. ${ }^{16}$ Thus, MIF produced by various cells appears to be involved in the enhancement of ocular inflammation and immune responses.

This work was partly supported by a grant in aid for scientific research from the Ministry of Education, Science, Sports and Culture of Japan; by a research grant for Behçet's Disease from the Ministry of Health and Welfare, Tokyo, Japan.

1 Bloom BR, Bennet B. Mechanism of a reaction in vitro ssociated with delayed-type hypersensitivity. Science 1966;153:80-2.

2 Bucala R. MIF rediscovered: cytokine, pituitary hormone, and glucocorticoid-induced regulator of the immune response. FASEB f 1996;10:1607-13.

3 Kitaichi N, Ogasawara K, Iwabuchi K, et al. Different influence of macrophage migration inhibitory factor (MIF) in signal transduction pathway of various $\mathrm{T}$ cell subsets. Immunobiology 2000;201:356-67.

4 Kitaichi N, Kotake S, Sasamoto Y, et al. Prominent increase of macrophage migration inhibitory factor in the sera of patients with uveitis. Invest Ophthalmol Vis Sci 1999;40: patients

5 Bernhagen J, Calandra T, Mitchell RA, et al. MIF is a pituitary-derived cytokine that potentiates lethal endotoxpituitary-derived cytokine that

6 Calandra T, Bernhagen J, Metz CN, et al. MIF as a glucocorticoid-induced modulator of cytokine production. Nature 1995;377:68-71.

7 De Vos AF, Hoekzema R, Kijlstra A. Cytokines and uveitis. Curr Eye Res 1992;11:581-97.

8 Kijlstra A. The role of cytokines in ocular inflammation. $\mathrm{Br}$ f Ophthalmol 1994;78:885-6.

9 Shimizu T, Abe R, Ohkawara A, et al. Macrophage migration inhibitory factor is an essential immunoregulatory cytokine in atopic dermatitis. Biochem biophys Res Commun 1997;240:173-8.

10 Rothova A, Buitenhuis HJ, Meenken C, et al. Uveitis and systemic disease. Br f Ophthalmol 1992;76:137-41.

11 Beckingsale AB, Davies J, Gibson JM, et al. Acute anterior uveitis, ankylosing spondylitis, back pain, and HLA-B27. Breitis, ankylosing spondylitis,

12 Matsuda A, Kotake S, Tagawa Y, et al. Detection and immunolocalization of macrophage migration inhibitory factor in rat iris and ciliary epithelium. Immunol Lett 1996;53:1-5. 
13 Nishihira J, Koyama Y, Mizue Y. Identification of macrophage migration inhibitory factor (MIF) in human vascular Cytokine 1998;10:199-205.

14 Shimizu T, Abe R, Ohkawara A, et al. Increased production of macrophage migration inhibitory factor by PBMCs of atopic dermatitis. $\mathcal{F}$ Allergy Clin Immunol 1999;104:65964 .
15 Bacher M, Metz CN, Calandra T, et al. An essential regulatory role for macrophage migration inhibitory factor in 54.

16 Kitaichi N, Matsuda A, Kotake S, et al. Inhibition of experimental autoimmune uveoretinitis with anti-macrophage migration inhibitory factor antibodies. Curr Eye Res 2000;20:109-14. 